\title{
ПЕРСПЕКТИВЫ ПОПУЛЯЦИОННОГО СКРИНИНГА РАКА ПРЕДСТАТЕЛЬНОЙ ЖЕЛЕЗЫ
}

\author{
АНТОН БАРЧУК
}

\begin{abstract}
Рак предстательной железы - одна из ключевых причин высокой смертности от рака в России и мире. При отсутствии эффективных методов профилактики для снижения смертности может быть перспективен популяционный скрининг с помощью простатического специифического антигена (ПСА). Несмотря на противоречивый характер рандомизированных исследований в нескольких работах показан реальный эффект скрининга по снижению смертности, достигающий $30 \%$. Рекомендации по популящионному внедрению данного метода резко ограничивает доля гипердиагностики, характерная для скрининга рака предстательной железы. В коротком обзоре литературы представлены возможные пути снижения гипердиагностики и повыщения популяционной эффективности скрининга рака предстательной железы. Представлень результаты анализа уже проведенных исследований, а также дизайн нового планируемого рандомизированного исследования.
\end{abstract}

Ключевые слова: рак предстательной железы, ПСА, скрининг, моделирование, рандомизированные исследования.

Рак предстательной железы постепенно становиться одной из главных причин смертей от онкологических заболеваний у мужчин пожилого возраста в России. К сожалению, методы первичной профилактики, т.е. элиминации факторов риска развития рака предстательной железы, не так эффективны в виду отсутствия какого-то одного доказанного значимого фактора развития этого заболевания. Предложенный много лет назад метод вторичной профилактики путем скрининга простатического специфического антигена (ПСА) в крови в теории обещал значимое снижение смертности. Скрининг рака предстательной железы имел все предпосылки занять ключевое место во вторичной профилактике рака, наряду с программами скрининга рака шейки матки, рака молочной железы и колоректального рака. Но на настоящий момент ни одна из крупных врачебных ассоциаций не рекомендует применение этого метода ранней диагностики. Несмотря на кажущуюся доступность и простоту, существуют несколько подводных камней при использовании данного метода в рамках популяционного скрининга. Основные проблемы связаны как раз с широтой применения данного метода в рамках популяционного скрининга.

До настоящего момента в мире было несколько рандомизированных исследований, где изучался скрининг рака предстательной железы. В далеком 2004 г. были опубликованы результаты исследования под авторством канадских ученых [Labrie et al. 2004]. B работе изучался скрининг с помощью ПСА, и несмотря на опубликованный результат (снижение смертности на 62\%), это исследование - отличный пример, как не следует анализировать рандомизированные исследования. АНТОН АЛЕКСЕЕВИч БАРчУК (barchuk.anton@gmail.com), УНИВЕРСИТЕТ ТАМПЕРЕ (ФИНЛЯНДИЯ), ФГБУ
«НМИЦ ОНКОЛОГИИ ИМ. Н.Н. ПЕТРОВА» МИНЗДРАВА РОССИИ (РОССИЯ).

СТАТЬЯ ПОСТУПИЛА В РЕДАКЦИЮ В ИЮЛЕ 2017 Г. 
Сначала были рандомизированы более 45000 мужчин в отношении 2:1. Но дальше исследование пошло не по плану его авторов. В группе ПСА-скрининга только 7000 из 30000 в итоге пришли на этот скрининг, а в группе контроля около 1500 из 15000 как раз выполнили исследование, хотя не должны были. Можно было бы сделать вывод, что исследование не состоялось из-за того, что большинство участников не следовало протоколу. Однако это не смутило канадских ученых, которые проигнорировали рандомизацию и просто сравнили тех, кто скрининг прошел, с теми, кто его не прошел, вне зависимости от группы рандомизации. За это они были подвергнуты жесткой критике коллег [Elwood 2004].

Ключевыми же исследованиями ПСА-скрининга в итоге стали американское PLCO (Prostate, Lung, Colorectal, and Ovarian Cancer Screening Trial) и европейское ERSCP (The European Randomized Study of Screening for Prostate Cancer). Причем, они были опубликованы одновременно в 2009 г. в «The New England Journal of Medicine» (NEJM) и вызвали бурю дискуссий. Все потому, что в публикации PLCO различий в смертности не было [Andriole et al. 2009], а в публикации ERSCP [Schröder et al. 2009] различия в смертности были. Казалось бы, два исследования с противоположными результатами, как так вышло и что теперь делать?

Прежде чем сравнивать PLCO и ERSPC, следует отметить, что рандомизированные исследования делят на «efficacy trials» и «effectivness trials». Довольно сложно перевести эти понятия, но это как исследования теоретической эффективности и исследования практической эффективности. Работает ли метод (лекарство) в теории и работает ли на практике, когда у нас помимо метода еще много других технических проблем, не связанных напрямую с чистой теоретической эффективностью метода [Ford, Norrie 2016]. При этом в реальности нет белого и черного, все исследования находятся где-то в промежутке от «efficacy» и «effectiveness».

Если вернуться к исследованиям ПСА-скрининга, то сразу бросается в глаза, что в американском исследовании PLCO был высокий уровень контаминации, т.е., как в канадском когда-то, мужчины, рандомизированные в группу ПСА, не делали ПСА, а в группе контроля, наоборот, делали, хотя не должны были. Ровно так, как и происходит в обычной жизни. По факту группы сравнения в PLCO были такие: в среднем 2,7 ПСА-тестов в среднем на одного пациента в ходе всего наблюдения в группе контроля против 5,0 ПСАтестов на каждого участника в группе скрининга. В итоге в PLCO сравнивался не эффект ПСА-скрининга, а эффект частоты выполнения этого самого скрининга. На эту темы выходит серия публикаций, уже начиная с 2010 г. [Pinsky et al. 2010]. Ко всему прочему, более $45 \%$ участников PLCO проходили ПСА-скрининг еще до включения в исследования, что тоже портит чистоту эксперимента. Собственно, в европейском ERSPC все было лишь несколько лучше. Еще в 2009 г. в публикации в журнале «European Urology» было показано, что в группе скрининга до теста добрались около 75\%, а в группе контроля $50 \%$ делали ПСА-тест при отсутствии симптомов и показаний [Roobol et al. 2009]. И несмотря на это, показано снижение смертности на 20\%. Начиная с 2009 г. было довольно много публикаций о коррекции контаминации в группах PLCO и ERSPC и о том, каков на самом деле эффект ПСА-скрининга на смертность. 
Чтобы понять различия в двух этих исследованиях, следует обратиться к крайне важной публикации сентября 2017 г. «Reconciling the Effects of Screening on Prostate Cancer Mortality in the ERSPC and PLCO Trials» [Tsodikov et al. 2017]. Следует отметить, что группа авторов публиковала и другие аналогичные статьи о скрининге. Например, статья о скрининге рака легкого 2014 г. была основой существующих американских рекомендаций по скринингу рака легкого USPSTF [De Koning et al. 2014]. Статьи 2014 и 2017 г. объединяет не только тема скрининга - они берут за основу моделирование эффектов скрининга по результатам рандомизированных исследований. Другими словами, обе статьи про то, как можно использовать данные существующих исследований, чтобы сделать выводы за пределами тех вопросов, которые эти исследования изначально ставили.

Также следует отметить, что эти статьи для широкого круга читателей, где представляется довольно популярная концепция моделирования. Тут ее использовали для того, чтобы подсчитать реальный эффект скрининга - «теоретическую эффективность». Также в статье широкой публике представляется довольно специфичный термин - MLT или «mean lead time». Чтобы понять, что это такое, надо знать теорию скрининга и варианты системных ошибок при оценке исследований скрининга.

Одна из причин, по которой нельзя оценить выживаемость в исследованиях скрининга - это «lead time bias» или «ошибка времени опережения». Концепция довольно проста: любая диагностика у бессимптомного человека приводит к тому, что мы передвигаем время установки диагноза заболевания на более ранний срок. При этом не факт, что мы изменяем судьбу пациента, т.е. спасаем его от смерти. Мы просто увеличиваем период времени от даты диагноза до даты смерти или выздоровления. Эффективный скрининг в отличие от неэффективного помимо увеличения этого периода («lead time») еще и предотвращает смерть. Существует теория, согласно которой эффективный скрининг предотвращает смерть из-за достаточно длинного «lead time» - времени опережения (рисунок 1).

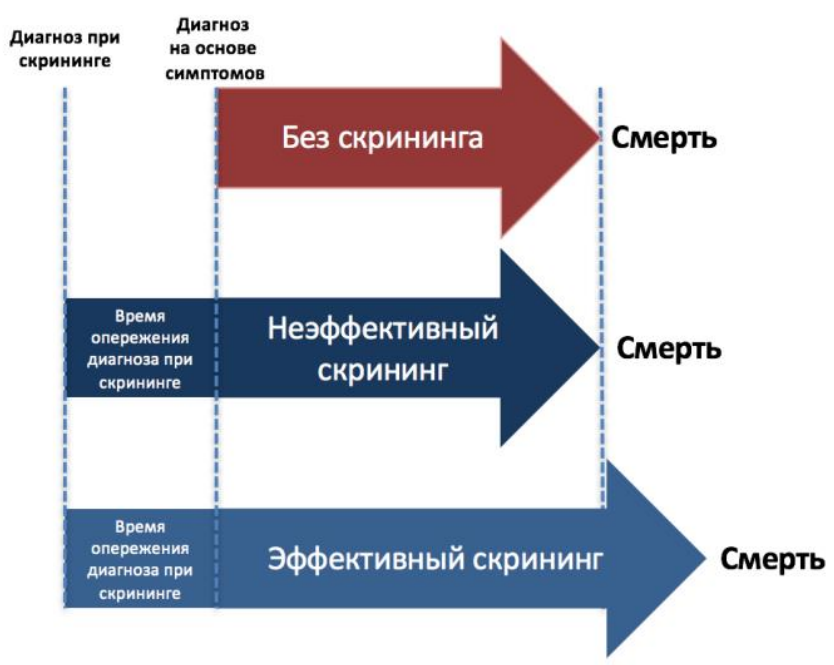

\section{Рисунок 1. Опережение времени постановки диагноза при эффективном и неэффективном скрининге (lead time)}

Источник: Адаптировано по [Evans et al. 2011]. 
Собственно, на этом и основаны математические модели, описанные в статье - они считают среднее время опережения установки «диагноза» в рандомизированных исследованиях в группах скрининга и контроля. Опережение диагноза в группе контроля в PLCO было намного больше, чем в ERSPC, как раз из-за поголовного скрининга в группе контроля. При этом в группе скрининга опережение диагноза было практически идентичным в PLCO и в ERSPC. Другими словами, оба исследования показали одно и тоже, никаких особых разногласий между ними нет. Далее авторы статьи использовали три математические модели (FHCRC, MISCAN и UMICH), настроили их на данные PLCO и ERSPC и посмотрели, как бы снижалась смертность в идеальном исследовании - она снижалась на те самые условные $30 \%$, известные нам еще в вышеупомянутой публикации 2009 г. [Roobol et al. 2009].

Можно ли теперь сказать, что скрининг рака простаты снижает смертность? Да, это факт, известный с 2009 г., и с ним мало кто спорит. Основные проблемы скрининга с помощью ПСА, к сожалению, лежат в других плоскостях. Одна из проблем в том, что несмотря на хороший относительный эффект, ПСА-скрининг конвертируется в весьма скромный абсолютный эффект, более чем скромный, так как смертность от рака предстательной железы крайне невысока после выявления опухоли. Но и это не главная проблема, основная - это гипердиагностика, которая может достигать 70\%. Об этом немало написано, не только в научных [Loeb et al. 2014], но и в научно-популярных публикациях. Главный вопрос сегодня: как эту проблемы решить.

Существует два способа решить проблемы ПСА-скрининга, чтобы изменить мнения и рекомендации большинства медицинских ассоциаций: увеличить абсолютный эффект и снизить гипердиагностику. Чтобы повысить абсолютный эффект, надо проводить скрининг среди тех, у кого риск умереть от рака простаты выше. Чтобы снизить гипердианостику, надо не проводить скрининг среди тех, у кого высокие шансы найти малоагрессивную, не мешающую жить опухоль.

В 2014 г. была опубликована еще одна работа, в которой моделируется скрининг на основании предыдущих рандомизированных исследований [Heijnsdijk et al. 2015]. В данном случае моделировали различные сценарии программ скрининга: в каком возрасте начать, в каком закончить, как часто выполнять анализ ПСА. Каждый сценарий приносил пользу в виде спасенных жизней, но и каждый приносил вред, связанный с гипердиагностикой.

Например, если начать скрининг в 55 лет и делать его каждый год до 75 лет, то на 1000 мужчин ПСА-скрининг обеспечит 6 лет жизни с учетом ее хорошего качества. При этом если закончить скрининг не в 75, а в 63 года, то выгоды будет в два раза больше (12 лет на 1000 мужчин), затрат при этом будет меньше. Почему так происходит? Просто ПСАскрининг портит жизнь пожилым мужчинам, у которых часто выявляются малоагрессивные опухоли. В итоге авторы пришли к выводу, что оптимальный скрининг следует начать в 55 лет, закончить в 59 и выполнять его раз в два года. То есть три теста ПСА за всю жизнь: в 55, 57 и 59 лет. Так можно решить проблему гипердиагностики в старшем возрасте - просто не выполнять скрининг после 59 лет.

Еще один способ решить проблему гипердиагностики - снизить инвазивность вмешательств при выявлении опухолей простаты. Исследования методов «активного 
наблюдения» выявленных с помощью ПСА опухолей предстательной железы заслуживают отдельного обзора, но одна публикация заслуживает внимания из-за тех же методов моделирования [De Carvalho et al. 2017]. На этот раз авторы попытались оценить влияние «активного наблюдения» опухолей простаты на смертность и излишнее лечение. Оказалось, что использование «активного наблюдения» с выполнением ежегодной биопсии увеличивает риск умереть от рака простаты на 1\%, но снижает долю ненужного лечения на 18\%. При выполнении биопсии раз в три года риск смерти от рака простаты повышался только на 2,3\%, доля ненужного лечения снижалась уже более чем на $30 \%$. Все эти результаты были получены для мужчин с опухолями низкого риска. Но даже у мужчин с опухолями среднего риска были получены аналогичные результаты: хоть выполнение наблюдения и повышало смертность на 2,7\%, но доля ненужного лечения снижалась на $23 \%$. Если объяснять это простым языком, мы сильно уменьшаем вред, не слишком сильно уменьшая пользу.

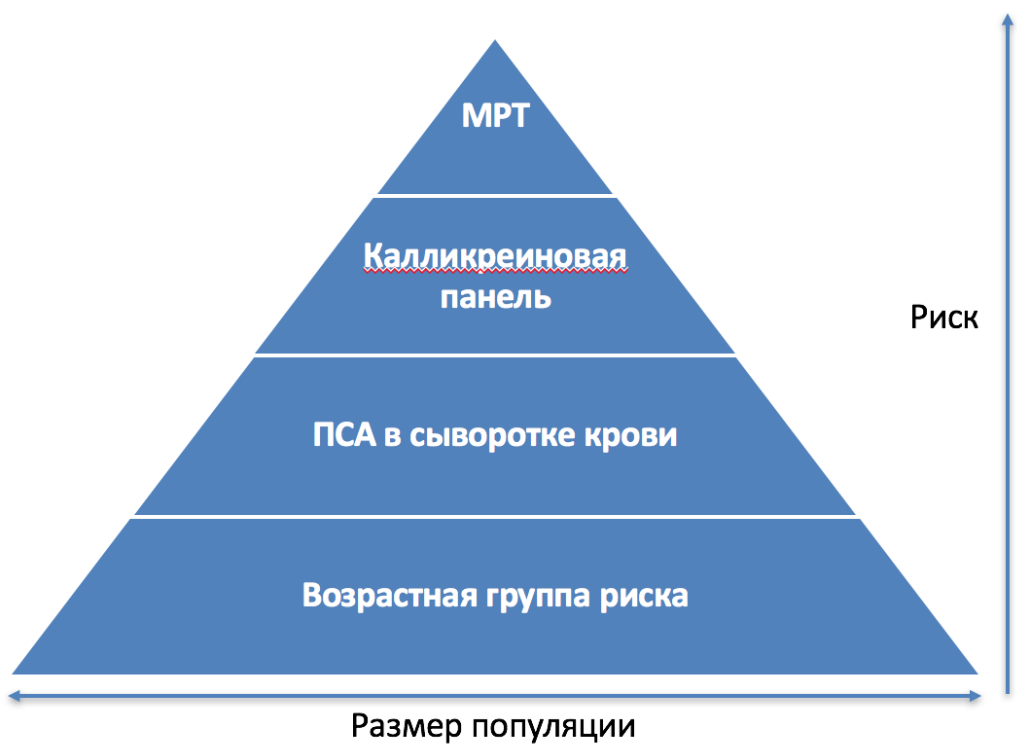

\section{Рисунок 2. Отбор пациентов на биопсию в исследовании ProScreen}

Источник: Адаптировано по [Auvinen et al. 2017].

Все эти способы влияют на гипердиагностику или долю ненужного лечения, но при этом мало влияют на абсолютный риск умереть от рака простаты. По этой причине следует отметить еще одну работу, посвященную скринингу рака предстательной железы. В июле было представлено новое рандомизированное исследование [Auvinen et al. 2017]. В нем ПСА-тест выступает уже не как метод выявления мужчин, которым показана биопсия, а лишь как первый этап в отборе мужчин с максимальным риском появление агрессивной опухоли. Предлагается довольно сложная модель скрининга: если ПСА повышен сильно, мужчина направляется на калликреиновый тест (4К-панель), если и этот анализ положительный, мужчина направляется на МРТ и только потом - на биопсию (рисунок 2). При этом в случае отрицательного результата на любом из этапов мужчина возвращается в обычный скрининг. Интервал этого скрининга зависит уже от того этапа, на котором с его простаты были сняты подозрения, и от первичного уровня ПСА. Несмотря на довольно сложную схему, это первые наметки персонализированного скрининга. К сожалению, 
первые результаты исследования мы получим лет через 15. Пока остается признать, что возможный вред от ПСА-скрининга превышает возможную пользу и решение о прохождение ПСА-теста следует все же принимать индивидуально, взвешивая заранее все индивидуальные риски.

\section{СОКРАЩЕНИЯ}

ПСА - Простатический специфический антиген

PLCO - Prostate, Lung, Colorectal, and Ovarian Cancer Screening Trial

ERSPC - The European Randomized Study of Screening for Prostate Cancer

\section{ЛИТЕРАТУРА}

Andriole G.L., E.D Crawford, R.L Grubb, S.S. Buys, D. Chia, T.R. Church et al. (2009). Mortality results from a randomized prostate-cancer screening trial // New England journal of medicine. 360(13): 1310-1319.

Auvinen A., A. Rannikko, K. Taari, P. Kujala, T. Mirtti, A. Kenttämies et al. (2017). A randomized trial of early detection of clinically significant prostate cancer (ProScreen): study design and rationale // European journal of epidemiology. 32(6): 521-527.

De Carvalho T.M., E.A. Heijnsdijk, H.J. de Koning (2017). Estimating the risks and benefits of active surveillance protocols for prostate cancer: a microsimulation study // BJU international. 119(4): 560-566.

De Koning H.J., R. Meza, S.K. Plevritis, K. ten Haaf, V.N. Munshi, J. Jeon et al. (2014). Benefits and harms of computed tomography lung cancer screening strategies: a comparative modeling study for the U.S. Preventive Services Task Force // Annals of internal medicine. 160(5): 311-320.

Elwood M. (2004). A misleading paper on prostate cancer screening // The prostate. 61(4): 372372.

Evans I., H. Thornton, I. Chalmers, P. Glasziou (2011). Testing treatments: better research for better healthcare. 2nd edition. London: Pinter \& Martin. 34 c.

Ford I., J. Norrie (2016). Pragmatic Trials // New England journal of medicine. 375(5): 454-463.

Heijnsdijk E.A.M., T.M. de Carvalho, A. Auvinen, M. Zappa, V. Nelen, M. Kwiatkowski et al. (2015). Cost-effectiveness of prostate cancer screening: a simulation study based on ERSPC data // Journal of the National cancer institute. 107(1): 366.

Labrie F., B. Candas, L. Cusan, J.L. Gomez, A. Bélanger, G. Brousseau et al. (2004). Screening decreases prostate cancer mortality: 11-year follow-up of the 1988 Quebec prospective randomized controlled trial // The prostate. 59(3): 311-318.

Loeb S., M.A. Bjurlin, J. Nicholson, T.L. Tammela, D.F. Penson, H.B. Carter et al. (2014). Overdiagnosis and overtreatment of prostate cancer // European urology. 65(6): 1046-1055.

Pinsky P.F., A. Black, B.S. Kramer, A. Miller, P.C. Prorok, C. Berg (2010). Assessing contamination and compliance in the prostate component of the Prostate, lung, colorectal, and ovarian (PLCO) cancer screening trial // Clinical trials. 7(4): 303-311. 
Roobol M.J., M. Kerkhof, F.H. Schröder, J. Cuzick, P. Sasieni, M. Hakama et al. (2009).

Prostate cancer mortality reduction by prostate-specific antigen-based screening adjusted for nonattendance and contamination in the European randomised study of screening for prostate cancer (ERSPC) // European urology. 56(4): 584-591.

Schröder F.H., J. Hugosson, M.J. Roobol, T.L.J. Tammela, S. Ciatto, V. Nelen et al. (2009). Screening and prostate-cancer mortality in a randomized European study // New England journal of medicine. 360(13): 1320-1328.

Tsodikov A., R. Gulati, E.A.M. Heijnsdijk, P.F. Pinsky, S.M. Moss, S. Qiu, et al. (2017). Reconciling the effects of screening on prostate cancer mortality in the ERSPC and PLCO trials // Annals of internal medicine. 167(7): 449-455. 


\title{
PERSPECTIVES ON POPULATION-BASED PROSTATE CANCER SCREENING
}

\section{$\underline{\underline{\text { ANTON BARCHUK }}}$}

\begin{abstract}
Prostate cancer is one of the key causes of high cancer mortality in Russia and the world.
In the absence of effective preventive measures, population-based screening using prostate-specific antigen (PSA) may be promising for mortality reduction. Despite conflicting results of randomized trials, the efficacy of prostate cancer screening in mortality reduction may be up to 30\%. Overdiagnosis, typical for prostate cancer screening, limits a population-based implementation. Possible methods to decrease the amount of overdiagnosis and increase the effectiveness of population-based prostate cancer screening are discussed in a brief review of the literature. An analysis of selected results of randomized trials and a design for a new randomized trial are presented.
\end{abstract}

Key words: prostate cancer, PSA, screening, modelling, randomized trials.

ANTON A. BARChUK (barchuk.anton@gmail.com), University of TAMPERE (FINLAND), N.N. Petrov NATIONAL CENTER OF ONCOLOGY (RUSSiA).

DATE RECEIVED: JULY 2017.

\section{REFERENCES}

Andriole G.L., E.D Crawford, R.L Grubb, S.S. Buys, D. Chia, T.R. Church et al. (2009). Mortality results from a randomized prostate-cancer screening trial // New England journal of medicine. 360(13): 1310-1319.

Auvinen A., A. Rannikko, K. Taari, P. Kujala, T. Mirtti, A. Kenttämies et al. (2017). A randomized trial of early detection of clinically significant prostate cancer (ProScreen): study design and rationale // European journal of epidemiology. 32(6): 521-527.

De Carvalho T.M., E.A. Heijnsdijk, H.J. de Koning (2017). Estimating the risks and benefits of active surveillance protocols for prostate cancer: a microsimulation study // BJU international. 119(4): 560-566.

De Koning H.J., R. Meza, S.K. Plevritis, K. ten Haaf, V.N. Munshi, J. Jeon et al. (2014). Benefits and harms of computed tomography lung cancer screening strategies: a comparative modeling study for the U.S. Preventive Services Task Force // Annals of internal medicine. 160(5): 311-320.

Elwood M. (2004). A misleading paper on prostate cancer screening // The prostate. 61(4): 372372.

Evans I., H. Thornton, I. Chalmers, P. Glasziou (2011). Testing treatments: better research for better healthcare. 2nd edition. London: Pinter \& Martin. 34 p.

Ford I., J. Norrie (2016). Pragmatic Trials // New England journal of medicine. 375(5): 454-463.

Heijnsdijk E.A.M., T.M. de Carvalho, A. Auvinen, M. Zappa, V. Nelen, M. Kwiatkowski et al. (2015). Cost-effectiveness of prostate cancer screening: a simulation study based on ERSPC data // Journal of the National cancer institute. 107(1): 366.

Labrie F., B. Candas, L. Cusan, J.L. Gomez, A. Bélanger, G. Brousseau et al. (2004). Screening decreases prostate cancer mortality: 11-year follow-up of the 1988 Quebec prospective randomized controlled trial // The prostate. 59(3): 311-318. 
Loeb S., M.A. Bjurlin, J. Nicholson, T.L. Tammela, D.F. Penson, H.B. Carter et al. (2014). Overdiagnosis and overtreatment of prostate cancer // European urology. 65(6): 1046-1055.

Pinsky P.F., A. Black, B.S. Kramer, A. Miller, P.C. Prorok, C. Berg (2010). Assessing contamination and compliance in the prostate component of the Prostate, lung, colorectal, and ovarian (PLCO) cancer screening trial // Clinical trials. 7(4): 303-311.

Roobol M.J., M. Kerkhof, F.H. Schröder, J. Cuzick, P. Sasieni, M. Hakama et al. (2009). Prostate cancer mortality reduction by prostate-specific antigen-based screening adjusted for nonattendance and contamination in the European randomised study of screening for prostate cancer (ERSPC) // European urology. 56(4): 584-591.

Schröder F.H., J. Hugosson, M.J. Roobol, T.L.J. Tammela, S. Ciatto, V. Nelen et al. (2009). Screening and prostate-cancer mortality in a randomized European study // New England journal of medicine. 360(13): 1320-1328.

Tsodikov A., R. Gulati, E.A.M. Heijnsdijk, P.F. Pinsky, S.M. Moss, S. Qiu et al. (2017). Reconciling the effects of screening on prostate cancer mortality in the ERSPC and PLCO trials // Annals of internal medicine. 167(7): 449-455. 